\section{Screening for abdominal aortic aneurysms was cost effective for prolonging survival from AAA related death in older men}

\author{
Multicentre Aneurysm Screening Study Group. Multicentre aneurysm screening study (MASS): cost effectiveness analysis of \\ screening for abdominal aortic aneurysms based on four year results from randomised controlled trial. BMJ \\ 2002;325:1135-8. \\ QUESTION: In older men, does ultrasonographic screening for abdominal aortic \\ aneurysms (AAA) sufficiently increase survival time free from AAA related death to make \\ it cost effective?
}

\section{Design}

4 year cost effectiveness analysis from a health service perspective of a randomised (allocation concealed*), blinded (data collectors and outcome assessors),* controlled trial $\}$.

\section{Setting}

4 centres in England, UK.

\section{Patients}

67800 men who were 65-74 years of age (mean age 69 y) and were identified from family physician and Health Authority patient lists. Family physicians excluded patients they considered unfit to be screened, including those with terminal illness, other serious health problems, or previous AAA repair. Mortality follow up was $99 \%$.

\section{Intervention}

Men were allocated to be invited ( $\mathrm{n}=33$ 839) or not to be invited ( $\mathrm{n}=33$ 961) for screening. The invited group received by mail an invitation from their family physician, an information booklet, and a questionnaire. Those accepting the invitation attended a clinic and had an ultrasonographic scan of the abdominal aorta. Scan results were sent to the family physicians. No contact was made with men in the uninvited group with respect to screening.

\section{Main cost and outcome measures}

Incremental cost effectiveness defined as the cost per year of life gained, survival, and healthcare resource use. Effects in life years were discounted at a rate of $1.5 \%$ and cost at $6 \%$. Costs were estimated in 2000 to 2001 British pounds.

\section{Main results}

Overall, mean costs per patient (relating to screening and to AAA related surgery in both groups) and the mean survival time free from death related to AAA were greater in the intervention group than in the control group (table). The corresponding incremental cost effectiveness ratio at 4 years was $£ 28400$ (95\% CI $£ 15000$ to $£ 146000$ ) per life year gained.

\section{Conclusion}

In older men, ultrasonographic screening for abdominal aortic aneurysms might be considered cost effective for increasing survival time free from death related to abdominal aortic aneurysms.

*See glossary. $\uparrow$ The Multicentre Aneurysm Screening Study Group. Lancet 2002;360:1531-9.

Invited $v$ not invited for abdominal aortic aneurysm screening at 4 years $\$$

\begin{tabular}{|c|c|c|c|}
\hline Outcomes & Invited & Not invited & Difference $(95 \% \mathrm{CI})$ \\
\hline $\begin{array}{l}\text { Mean costs }(£) \text { after adjustment for } \\
\text { censoring and discounting at } 6 \% / \text { year }\end{array}$ & 98.42 & 35.03 & 63.39 (53.31 to 73.48$) \S$ \\
\hline $\begin{array}{l}\text { Mean survival time (d) after discounting } \\
\text { at } 1.5 \% / \text { year }\end{array}$ & 1394.73 & 1393.92 & $0.82(0.16$ to 1.47$) \S$ \\
\hline
\end{tabular}

§Outcomes are significantly greater in the invited group.

\section{COMMENTARY}

AAA is potentially an ideal candidate for screening because the condition frequently remains asymptomatic until rupture with catastrophic results. The results of the clinical trial by the Multicentre Aneurysm Screening Study Group ${ }^{1}$ suggest that screening is beneficial. However, a question remains: do the relatively small benefits seen in the trial $(<1$ additional day of life per patient screened) justify the net costs of screening?

The large and high quality study by the Multicentre Aneurysm Screening Study Group suggests that even with the relatively short time horizon of 4 years, AAA screening falls within the usual bounds of acceptable cost effectiveness. Extrapolation to a longer time horizon makes the argument even more convincing. As with all screening, the cost effectiveness depends on context. This study was done in the UK National Health Service, and those wishing to apply the results in other settings would need to consider the effect of differences in disease incidence, the incidental pick up rate, and differences in investigation and treatment costs. The sensitivity analysis in this study suggested that screening costs have a substantial effect on overall cost effectiveness. It is also necessary to consider the implications in terms of the capacity of the health system to deal with the additional investigations and treatments that would become necessary and any probable opportunity costs in relation to displaced activity.

Despite these considerations, screening for AAA seems to be cost effective. However, questions remain about the details of screening programmes, such as the applicability of screening to women, the best age at first screening, the need and appropriate intervals for rescreening, and the further management of small and borderline aneurysms identified by screening. The situation may be further altered by the new technique of endovascular aneurysm repair, ${ }^{2}$ which may be particularly applicable to small AAAs identified through screening. If ongoing clinical trials show the technique to have net safety benefits, then this may further alter the balance in favour of screening. Jonathan A Michaels, MA, MChir, FRCS Sheffield Teaching Hospitals NHS Trust Sheffield, UK

1 The Multicentre Aneurysm Screening Study Group. The Multicentre Aneurysm Screening Study (MASS) into the effect of abdominal aortic aneurysm screening on mortality in ing Study (MASS) into the effect of abdominal aortic aneurysm screening on mortality in

2 Adriaensen ME, Bosch JL, Halpern EF, et al. Elective endovascular versus open surgical repair of abdominal aortic aneurysms: systematic review of short-term results. Radiology 2002;224:739-7. 